\title{
3 Research Square \\ Free cortisol/cortisone ratio in morning urine as a biomarker of health status in older Japanese adults
}

\section{Akiko Mutsamoto ( $\square$ matsumoa@cc.saga-u.ac.jp )}

Saga University School of Medicine https://orcid.org/0000-0002-8445-4071

\section{Chisato Shimanoe}

Faculty of Medicine, Saga University

\section{Megumi Hara}

Saga University https://orcid.org/0000-0002-8708-3237

\section{Chiho Akao}

Saga University School of Medicine

\section{Yuichiro Nishida}

Saga University https://orcid.org/0000-0003-2320-7234

\section{Masayoshi Ichiba}

Saga University School of Medicine

\section{Keitaro Tanaka}

Faculty of Medicine, Saga University https://orcid.org/0000-0002-5725-4736

\section{Article}

Keywords: cortisol, cortisol/cortisone ratio, urine, liquid chromatography-mass spectrometry

Posted Date: July 22nd, 2020

DOI: https://doi.org/10.21203/rs.3.rs-41343/v1

License: (c) (i) This work is licensed under a Creative Commons Attribution 4.0 International License. Read Full License 


\section{Abstract}

Secretion of cortisol in humans peaks in the morning, reflecting robust circadian rhythms, which are essential for homeostasis. To examine the hypothesis that free cortisol levels in the morning can predict general health status, free cortisol and cortisone in urine samples collected from 7813 Japanese older adults upon waking were measured by liquid chromatography-mass spectrometry (LCMS). The ratio of cortisol to cortisone in 5298 urine collected between 6 and 11 AM showed consistent correlations with favorable personal attributes linking to good health. The odds ratio of poor health, as defined by multiple clinical criteria (BMI $\geq 25, S B P \geq 140 \mathrm{mmHg}, \mathrm{C}$ reactive protein $\geq 0.5 \mathrm{mg} / \mathrm{dL}$, and $\mathrm{HbA} 1 \mathrm{c} \geq 5.6 \%$ ) or cardio-cerebro-vascular disease history, robustly showed negative associations with the cortisol/cortisone ratio. The free cortisol/cortisone ratio in urine samples collected upon waking is a promising biomarker of good health and might be applicable to a wide range of research and medical settings.

\section{Introduction}

Biomarkers of the stress response, such as catecholamines and glucocorticoids, are often used as indicators of exposure to harmful factors, such as environmental toxins and harsh physical elements ${ }^{1-3}$. The stress response itself is not harmful and is essentially a protective reaction to maintain homeostasis.

Stress responsiveness is strongly influenced by circadian rhythms, which regulate important biological functions, such as immunity, cognition, circulation, and energy metabolism ${ }^{4,5}$. The stress response is triggered by various factors, such as exercise ${ }^{6,7}$, changes in temperature ${ }^{8-10}$, and psychological stress ${ }^{11}$. If these events are transient, they may be beneficial ${ }^{5,12}$ and contribute to the maintenance of a robust stress response and circadian rhythm stability ${ }^{13,14}$. However, long-term stress, such as aging ${ }^{7}$ and mental trauma ${ }^{15,16}$, lowers cortisolresponsiveness (reduced spike amplitude), leading to disruption of circadian rhythms ${ }^{17}$.

We hypothesize that high cortisol secretion in the morning reflects stress responsiveness and stability of circadian rhythms and is therefore a potential marker for good health. To evaluate this hypothesis, we conducted a cross-sectional study of a general cohort of older Japanese adults.

While cortisol is commonly measured in a variety of biological samples, we focused on analyzing cortisol in urine. Blood cortisol shows a pulsatile secretion pattern reflecting an ultradian rhythm and thus, its levels change greatly over time ${ }^{8}$. Therefore, the average concentration of cortisol in blood cannot be determined by a single measurement. Saliva also reflects blood cortisol levels ${ }^{18}$ and has similar limitations. With urine, it is possible to obtain the average concentration over a fixed period of time. Although hair and nails also have the advantage of determining long-term cortisol levels, the measurement protocol in these tissues is not simple and therefore unsuitable for large-scale studies.

We have developed a large-scale strategy for the measurement of free glucocorticoids by liquid chromatographymass spectrometry (LCMS) ${ }^{19}$. In the present study, we analyzed free glucocorticoids using strict quality control measures and carefully validated the data. We then examined the association between cortisol markers and health status, with the ultimate aim of identifying an effective biomarker for overall health.

\section{Materials And Methods}




\section{Subjects and sample collection}

A second survey of the Japanese Multi-Institutional Collaborative Cohort in Saga district was conducted for two years from $2010^{20}$. This study included 8454 participants, aged 45 to 74 years, who were asked to provide urine samples taken immediately upon waking. The samples were obtained and stored frozen at $-80^{\circ} \mathrm{C}$ until the time of analysis. Urinary creatinine concentration was measured as described previously ${ }^{21}$ and additional information, including physical and clinical data and medical history, was obtained from questionnaires and by examination on-site. Regular physical activity was assessed using an accelerometer (Life-coder, Suzuken Co. Ltd Nagoya, Japan) for 10 days as described previously ${ }^{22}$. This study was approved by the Ethics Committee of Saga University School of Medicine.

\section{LCMS analysis and data processing}

Of 8454 samples, 641 were not analyzed because of unavailability of the equipment or lack of a urine specimen. The $\mathrm{pH}$-adjusted urine spiked with internal standards (IS) (cortisol-d4) was pretreated on a C18 solid-phase extraction (SPE) column, further separated on a $\mathrm{C} 18$ analysis column, and then analyzed by mass spectrometry. To minimize measurement error due to a matrix effect (alteration of ionization efficiency of target analytes in the presence of co-eluting compounds on the same matrix), the standard solution was diluted with a water extract from the SPE column of the same type. The detailed protocol is described elsewhere ${ }^{19}$.

The sensitivity of the mass spectrometer fluctuated during the 15 months of analysis; therefore, the following quality control was performed. (1) A standard curve was drawn by the area ratio (target/cortisol-d4) of eight levels of standards before and after the measurement of 30 unknown samples (total of 46 samples $=$ one batch). (2) Three types of standard curves were drawn for the low concentration range $(-10 \mathrm{ng} / \mathrm{mL})$, medium concentration range $(-100 \mathrm{ng} / \mathrm{mL})$, and high concentration range $(-300 \mathrm{ng} / \mathrm{mL})$. The lower limit of detection (LOD) was determined for each batch (three times the standard deviation of the difference between the expected concentration and the actual value in the low concentration range). (3) Because the area of IS of the unknown sample was smaller than that of the standards (119463 vs. 243747 as medians), presumably affected by the extraction efficiency and matrix effect, the LOD for the individual unknown sample (LODi) was further determined based on the IS level of the unknown sample and that of the standards of the corresponding batch (median value). For example, if the LOD of a batch was $0.3 \mathrm{ng} / \mathrm{mL}$ and the IS area in an unknown sample was half the area of the standards, the LODi was set at $0.3 / 0.5=0.6 \mathrm{ng} / \mathrm{mL}$. If the value was below the LODi, half the LODi value was applied (as in 69 samples of cortisol and 15 samples of cortisone). If the value was above the range of the standard curve, the estimated value was applied.

\section{Data exclusion due to measurement failure}

During the analysis of 7813 samples in 261 batches, a total of 613 samples (7.8\%) were excluded (Fig. 1).

Twelve batches were inaccurate according to the criteria we defined, namely, one or more correlation coefficients of the calibration curves in a batch were $<0.9$, and two batches with low LOD (either cortisol or cortisone was $\geq 2.5 \mathrm{ng} / \mathrm{mL}$ ) were excluded. The 65 samples $(0.8 \%)$ with a cortisol LODi $\geq 5 \mathrm{ng} / \mathrm{mL}(0.8 \%)$ were also excluded. All of the cortisone LODi were considered sufficient $(<10 \mathrm{ng} / \mathrm{mL})$. We also excluded 130 specimens, in which cortisol could not be identified because of unknown interference peaks.

\section{Urinary thickness adjustment}


To correct for the thickness of urine, a creatinine-adjustment is generally applied; however, there is a major limitation of creatinine adjustments, namely, susceptibility to age, sex, and race ${ }^{23}$; therefore, we used another internal standard, cortisone, the inactive form of cortisol (cortisol/cortisone ratio). When both cortisol and cortisone were below the LODi, the cortisol/cortisone ratio was regarded as a missing value (14 samples).

\section{Statistical analysis}

The statistical software SAS 9.4 (SAS Institute Inc., Cary, NC, USA.) was used to determine statistical significance. In regression analyses, a Bonferroni correction a for the replication was set at $0.025(0.05 / 2)$ because two types of markers were tested. Odds ratio (OR) was estimated using logistic regression analysis, where a Bonferroni correction a for the replication was set at $0.0125(0.05 / 4)$ because four types of outcomes were tested.

\section{Results}

\section{Measurement accuracy}

To ensure that our measurements were consistent, we carefully monitored calibration curves and internal standards throughout the study, and excluded data with insufficient accuracy. During the analysis of 7813 samples in 261 batches, where a batch is defined as a measurement group sharing calculation curves with 30 or less unknown samples, 12 batches including 418 unknown samples plus 195 unknown samples in other batches, were excluded as shown in Fig. 1. Table S1 presents the quality of the standard curves in 247 batches. The standard curves demonstrate that our measurements were accurate throughout the study; the correlation coefficient (median, range) of the calibration curve was $>0.99(0.90-1.00)$, and the detection limits (median, range) for cortisol and cortisone were $0.33(0.01-2.0) \mathrm{ng} / \mathrm{mL}$ and $0.55(0.02-2.3) \mathrm{ng} / \mathrm{mL}$, respectively.

\section{Data distribution}

Distribution of subjects by sex, age and time of urine collection was shown in Table S2. The median values (interquartile range) of cortisol, cortisone, and creatinine were $19.4(11.4-32.7) \mathrm{ng} / \mathrm{mL}, 57.3(38.2-85.0) \mathrm{ng} / \mathrm{mL}$, and $0.82(0.53-1.27) \mathrm{mg} / \mathrm{mL}$, respectively. Histograms and box plots of cortisol levels separated by urinary collection times are shown in Figure 2 . The cortisol values differed greatly by collection time; urine collected from 7 to 9 AM showed the highest values for cortisol (Figure 2A), while cortisone showed less variation over time, especially from 6 to 11 AM (Figure 2B). Creatinine was also relatively high in the morning (Figure 2C). Both creatinine-adjusted cortisol and the cortisol/cortisone ratio exhibited no significant variation between 6 and 11 AM (Figures 2D, 2E). We therefore used urine samples collected between 6 and 11 AM $(N=5298)$ for association analysis. Distribution of the urine markers in this sub cohort is shown in Table 1.

\section{Association between free cortisol levels in morning urine and health status}

We hypothesized that high cortisol levels in morning urine would be a sign of good health. To test this hypothesis, we first performed rank correlation analyses between the cortisol levels in urine samples collected between 6 and 11 AM $(N=5298)$ and the characteristics of participants.

The associations we identified were similar between creatinine-adjusted cortisol and the cortisol/cortisone ratio. However, creatinine-adjusted cortisol exhibited some results contrary to our hypothesis (positive associations with old age and cold weather, and no association with systolic blood pressure (SBP) or hemoglobin A1C 
(HbA1c). Conversely, the cortisol/cortisone ratio consistently showed a strong association with female sex, youth, warm weather, regular sleep, less smoking/drinking, physical activity, lower SBP, lower BMI, lower HbA1c, and lower CRP (Table 2). Unadjusted cortisol also showed a similar tendency, but some results were contrary to our hypothesis (high in men, no association with season, smoking or SBP) (Table S3).

Additionally, the following discrepancies were observed (Tables 2 and S3). First, urinary creatinine levels were positively associated with unadjusted-cortisol and the cortisol/cortisone ratio, but negatively associated with creatinine-adjusted cortisol. Second, menopause was associated with lower unadjusted-cortisol and higher creatinine-adjusted cortisol levels, but no association was found with the cortisol/cortisone ratio. Third, corticosteroids showed a negative association with unadjusted-cortisol and creatinine-adjusted cortisol, but no correlation was found with the cortisol/cortisone ratio. Fourth, a significant relationship with perceived stress was observed only with the cortisol/cortisone ratio.

Some of the relationships mentioned above were not significant after adjusting for gender and age (Table S4). $\mathrm{BMI}$ and CRP were found to have a strong and stable association with all cortisol markers.

\section{Association between cortisol markers and odds ratio of being unhealthy}

To further examine whether free cortisol levels in morning urine predicts health status, cortisol levels were divided into tertiles (low, medium, high), and the odds ratio (OR) of some health indicators was estimated by tertile. The characteristics of participants by tertile are shown in Table S5.

As shown in Table 3 (crude model), the OR of poor health defined by the following criteria, BMI $\geq 25, \mathrm{SBP} \geq 140$ $\mathrm{mmHg}, \mathrm{CRP} \geq 0.5 \mathrm{mg} / \mathrm{dL}$, and $\mathrm{HbA} 1 \mathrm{c} \geq 5.6 \%$, was higher in participants with lower cortisol levels, although the cortisol/cortisone ratio showed a stronger association (regression coefficient for an increase per tertile $(B)=$ -0.38 with $p<0.0001$ when defined by 3 findings, and $B=-0.66$ with $p<0.0001$ when defined by 4 findings), than creatinine-adjusted cortisol $(B=-0.18$ with $p=0.0009$ for 3 findings, and $B=-0.29$ with $p=0.0152$ for 4 findings). Those associations were retained after adjusting for sex, age, smoking and drinking, physical activity, use of corticosteroids, and perceived stress (Table 3, model 1). The association of the OR with the cortisol/cortisone ratio was nearly maintained after stratified analysis by sex and age; however, the association observed for creatinine-adjusted cortisol was significantly weakened (Tables S6 and S7).

Next, history of cardio- and cerebrovascular disease (CCVD) was defined as an indicator of poor health because most CCVD patients have irreversible pathologies, such as arteriosclerosis. As expected, the OR of CCVD history was higher in subjects with low cortisol (B and $p$ values for creatinine-adjusted cortisol were -0.26 and 0.0005 , respectively, and for the cortisol/cortisone ratio were -0.37 and $<0.0001$, respectively; Table 2 , crude model). Again, stratification by sex and age strongly reduced the association with creatinine-adjusted cortisol, but the association with the cortisol/cortisone ratio was maintained (Tables S6 and S7). The multi-adjusted OR, as in model 1, still showed a negative association with cortisol levels ( $B$ and $p$ values for creatinine-adjusted cortisol were -0.24 and 0.0018 , respectively, and for cortisol/cortisone were -0.27 and 0.0004 , respectively) (Table 3 , model 1). Moreover, after further adjustments for SBP, BMI, HbA1c and CRP, the negative trend was still significant (B and $\mathrm{p}$ values for creatinine-adjusted cortisol were -0.22 and 0.0057 , respectively, and for cortisol/cortisone were -0.22 and 0.0065 , respectively; Table 3, model 2), which suggests an additional relationship between CCVD history and cortisol levels that cannot be explained by the lifestyle and laboratory findings. 
Malignancies are unlikely to affect health after treatment. As hypothesized, there was no association between the OR of malignancies and cortisol levels (Tables 3, S6 and S7).

\section{Discussion}

The urinary concentration of free cortisol and cortisone in 7200 older Japanese adults was analyzed, and these values were consistent with previous reports ${ }^{24-26}$. Cortisol was high in urine collected in the morning, as expected, and the corrected values (creatinine-adjusted cortisol and cortisol/cortisone ratio) showed little variation over time between 6 and 11 AM ( $n=5298)$. In 5298 samples, the cortisol/cortisol ratio consistently showed strong associations with indicators of good health, whereas creatinine-adjusted cortisol levels did not. Furthermore, the OR of being unhealthy, defined by a BMI $\geq 25$, systolic blood pressure $\geq 140 \mathrm{mmHg}, \mathrm{CRP} \geq$ $0.5 \mathrm{mg} / \mathrm{dL}$, and $\mathrm{HbA} 1 \mathrm{c} \geq 5.6 \%$, or a history of CCVD, was higher in subjects with lower cortisol levels. The association was robust with the cortisol/cortisol ratio, but not with creatinine-adjusted cortisol. Therefore, our study indicates that the level of free cortisol/cortisone ratio in morning urine might be a good biomarker for general health.

A major strength of the present study is its precise, large-scale measurement of free cortisol by LCMS. This level of accuracy in free cortisol and cortisone levels at such a scale has never been reported. A widely-used method for quantifying cortisol, enzyme-linked immunosorbent assay (ELISA), is limited because the measurement of active free cortisol and cortisone is compromised ${ }^{27,28}$. Urinary metabolites of cortisol, such as sulfate and glucuronide conjugates and other compounds with similar structures, may also be detected using ELISA, which decreases the specificity of the assay. Another strength of our protocol is the simultaneous measurement of cortisol and cortisone, reducing possible errors which could occur if analyzed separately.

There are several possible reasons why the cortisol/cortisone ratio represents a better correlation to good health than creatinine-adjusted cortisol alone. First, the variation in baseline levels in cortisol among individuals could be corrected by taking the ratio, because cortisol and cortisone levels are strongly correlated $(R=0.84 ;$ Figure S1) (as reference, the cortisol correlates creatinine weakly with $\mathrm{R}=0.32$; Figure $\mathrm{S} 2$ ). For example, corticosteroid use typically affects an individual's baseline level of cortisol by inhibiting endogenous levels of cortisol ${ }^{29}$. In fact, corticosteroid reduces both cortisol and cortisone levels (Table S3); therefore, the cortisol/cortisone ratio is maintained.

Second, a mechanistic shift caused by prolonged stress might explain the strong association of the cortisol/cortisone ratio to good health. Healthy, pulsatile secretion of cortisol that occurs during the stress response and/or because of circadian rhythms is stimulated predominantly by hypothalamic corticotropinreleasing hormone $(\mathrm{CRH})$, but also by arginine vasopressin (AVP), followed by pituitary adrenocorticotropic hormone $(\mathrm{ACTH})$ release. ACTH stimulation increases cortisol but not cortisone, resulting in an increase in the cortisol/cortisone ratio ${ }^{30}$. However, this mechanism of cortisol regulation changes when the stress response is prolonged and homeostasis is threatened; $\mathrm{CRH}$ expression is suppressed under these conditions, and AVP increases and becomes the predominant regulator of cortisol ${ }^{31,32}$. AVP activates $11 \beta$-hydroxysteroid dehydrogenase type $2(11 \beta-H S D 2){ }^{33}$, which converts cortisol to cortisone to protect the mineralocorticoid receptor from activation by cortisol, presumably to inhibit an unnecessary rise in blood pressure caused by the increase in AVP. Thus, when the stress response is prolonged, the cortisol/cortisone ratio may decrease because of this mechanistic shift in cortisol regulation. 
Third, the weak association of creatinine-adjusted cortisol to good health could be explained by the creatinine correction. The present study showed that urinary creatinine levels were low in women $(p<0.0001)$ and elderly people ( $p$ for trend $<0.0001$ ) (Table 1 ), as reported elsewhere ${ }^{23}$; therefore, with the correction, creatinine-adjusted cortisol levels in elderly women appear high (Table 1). A positive relationship between creatinine-adjusted cortisol and menopausal status $(R=0.12, p<0.0001$, Table 2$)$ could be explained by confounding factors, such as age; in fact, adjusting for age dramatically weakened this association $(R=0.03, p=0.054$, Table $S 4)$.

Whether activation of $11 \beta$-hydroxysteroid dehydrogenase type 1 (11ß-HSD1), which is expressed in peripheral tissues, such as adipose tissue and liver, affects the cortisol/cortisone ratio is an important question because $11 \beta$-HSD1 converts cortisone into cortisol. Because activation of 11ß-HSD1 is associated with obesity, metabolic disorders, and chronic inflammation ${ }^{34-36}$, poor health seems to increase $11 \beta$-HSD1 activity and the amount of cortisol in adipose tissue and liver. However, this is inconsistent with our hypothesis that the cortisol/cortisone ratio is associated with good health. Still, as seen in a previous report that shows no association between hypercortisolemia and obesity or rheumatoid arthritis ${ }^{37,38}$, tissue-specific $11 \beta$-HSD 1 activation unlikely increases systemic cortisol levels. While healthy cortisol release is pulsatile, 11ß-HSD1-induced cortisol is constant and flat. This chronic and consistent release of cortisol could disturb homeostasis ${ }^{5}$, and possibly circadian rhythms, resulting in low cortisol in morning urine.

To date, cortisol has been mainly used as a biomarker in various epidemiological and experimental studies. First, it has been used to estimate exposure levels to harmful factors by testing for healthy biological responses to harmful stressors ${ }^{39,40}$. Second, it has also been used to determine health status by testing for cortisol reactivity. This is typically done by measuring cortisol before and after stress intervention, before and after waking up 16,41, or by comparison with a control group in an experimental setting ${ }^{42}$. This study proposes a novel biomarker for good health based on the assumption that good health correlates with robust circadian rhythms and high levels of cortisol in the morning. However, we did not measure the peak-to-trough amplitude of the cortisol rhythm in our study. Previous reports indicate a positive association between peak levels of cortisol and circadian rhythm amplitude ${ }^{17,41}$, but this relationship needs to be verified.

Another limitation of our study is that it only included older Japanese adults living in a specific region of Japan. Our findings should be validated in additional Japanese populations and populations outside of Japan.

Nevertheless, we believe our findings are significant and may be relevant to the general population. Because urine samples are quite stable ( 24 hours at room temperature) ${ }^{19}$ and because we have described our methodology in great detail, we hope that this protocol can be easily adopted and widely used.

In conclusion, using a simple and highly accurate LCMS method, we measured free cortisol and cortisone in more than 7000 urine samples obtained from older Japanese residents. The free cortisol/cortisone ratio in morning urine was found to be a good biomarker for overall health and could be applied to various research and clinical settings.

\section{Declarations}

\section{Acknowledgments}


This work was supported by JSPS KAKENHI (18K10049, 16H06277, 15K01718, 221S0001, and 17015018), and the Ministry of Internal Affairs and Communications, Japan (JPMI10001). We thank Mikako Horita for helping experiments, and Edanz Group (https://en-author-services.edanzgroup.com/) for editing a draft of this manuscript.

\section{Author contributions}

A.M., C.S. and M.H. designed the study, A.M., C.M. and C.A. performed experiments and data analysis, A.M. wrote the manuscript, and M.H., Y.N., M.I. and K.T. revised the manuscript.

All participants consented to participate in the study, and also to publish achievements, by signing a consent form.

\section{References}

1. Selander, J. et al. Saliva cortisol and exposure to aircraft noise in six European countries. Environ Health Perspect 117, 1713-1717, doi:10.1289/ehp.0900933 (2009).

2. Bechshoft, T. O. et al. Measuring environmental stress in East Greenland polar bears, 1892-1927 and 19882009: what does hair cortisol tell us? Environ Int 45, 15-21, doi:10.1016/j.envint.2012.04.005 (2012).

3. Hajat, A. et al. The Association between Long-Term Air Pollution and Urinary Catecholamines: Evidence from the Multi-Ethnic Study of Atherosclerosis. Environ Health Perspect 127, 57007, doi:10.1289/EHP3286 (2019).

4. Ly, J. Q. M. et al. Circadian regulation of human cortical excitability. Nat Commun 7, 11828, doi:10.1038/ncomms11828 (2016).

5. Russell, G. \& Lightman, S. The human stress response. Nat Rev Endocrinol 15, 525-534, doi:10.1038/s41574-019-0228-0 (2019).

6. Starks, M. A., Starks, S. L., Kingsley, M., Purpura, M. \& Jager, R. The effects of phosphatidylserine on endocrine response to moderate intensity exercise. J Int Soc Sports Nutr 5, 11, doi:10.1186/1550-2783-5-11 (2008).

7. Traustadottir, T., Bosch, P. R., Cantu, T. \& Matt, K. S. Hypothalamic-pituitary-adrenal axis response and recovery from high-intensity exercise in women: effects of aging and fitness. The Journal of clinical endocrinology and metabolism 89, 3248-3254, doi:10.1210/jc.2003-031713 (2004).

8. Cameron, A. et al. Temperature-responsive release of cortisol from its binding globulin: a protein thermocouple. The Journal of clinical endocrinology and metabolism 95, 4689-4695, doi:10.1210/jc.20100942 (2010).

9. Rotllant, J., Balm, P. H., Wendelaar-Bonga, S. E., Perez-Sanchez, J. \& Tort, L. A drop in ambient temperature results in a transient reduction of interrenal ACTH responsiveness in the gilthead sea bream (Sparus aurata, L.). Fish Physiology and Biochemistry 23, 265-273, doi:https://doi.org/10.1023/A:1007873811975 (2000).

10. Follenius, M., Brandenberger, G., Oyono, S. \& Candas, V. Cortisol as a sensitive index of heat-intolerance. Physiol Behav 29, 509-513, doi:10.1016/0031-9384(82)90274-8 (1982).

11. Rotllant, J. et al. Pituitary proopiomelanocortin-derived peptides and hypothalamus-pituitary-interrenal axis activity in gilthead sea bream (Sparus aurata) during prolonged crowding stress: differential regulation of 
adrenocorticotropin hormone and alpha-melanocyte-stimulating hormone release by corticotropin-releasing hormone and thyrotropin-releasing hormone. Gen Comp Endocrinol 119, 152-163, doi:10.1006/gcen.2000.7508 (2000).

12. McEwen, B. S. Stress, adaptation, and disease. Allostasis and allostatic load. Annals of the New York Academy of Sciences 840, 33-44 (1998).

13. Drogos, L. L. et al. Aerobic exercise increases cortisol awakening response in older adults. Psychoneuroendocrinology 103, 241-248, doi:10.1016/j.psyneuen.2019.01.012 (2019).

14. Tortosa-Martinez, J., Manchado, C., Cortell-Tormo, J. M. \& Chulvi-Medrano, I. Exercise, the diurnal cycle of cortisol and cognitive impairment in older adults. Neurobiol Stress 9, 40-47, doi:10.1016/j.ynstr.2018.08.004 (2018).

15. Carpenter, L. L. et al. Decreased adrenocorticotropic hormone and cortisol responses to stress in healthy adults reporting significant childhood maltreatment. Biological psychiatry 62, 1080-1087, doi:10.1016/j.biopsych.2007.05.002 (2007).

16. Houtepen, L. C. et al. Genome-wide DNA methylation levels and altered cortisol stress reactivity following childhood trauma in humans. Nat Commun 7, 10967, doi:10.1038/ncomms10967 (2016).

17. Feng, X. et al. Maternal separation produces lasting changes in cortisol and behavior in rhesus monkeys. Proc Natl Acad Sci U S A 108, 14312-14317, doi:10.1073/pnas.1010943108 (2011).

18. Crewther, B. T., Obminski, Z., Orysiak, J. \& Al-Dujaili, E. A. S. The utility of salivary testosterone and cortisol concentration measures for assessing the stress responses of junior athletes during a sporting competition. J Clin Lab Anal 32, doi:10.1002/jcla.22197 (2018).

19. Matsumoto, A., Shimanoe, C., Tanaka, K., Ichiba, M. \& Hara, M. Development of suitable method for largescale urinary glucocorticoid analysis by liquid chromatography-mass spectrometry. Journal of chromatography. B, Analytical technologies in the biomedical and life sciences 1057, 62-69, doi:10.1016/j.jchromb.2017.04.031 (2017).

20. Hara, M. et al. Factors associated with non-participation in a face-to-face second survey conducted 5 years after the baseline survey. J Epidemio/ 25, 117-125, doi:10.2188/jea.JE20140116 (2015).

21. Hara, M. et al. Intensity-specific effect of physical activity on urinary levels of 8-hydroxydeoxyguanosine in middle-aged Japanese. Cancer Sci 107, 1653-1659, doi:10.1111/cas.13070 (2016).

22. Nishida, Y. et al. Objectively measured physical activity and inflammatory cytokine levels in middle-aged Japanese people. Prev Med 64, 81-87, doi:10.1016/j.ypmed.2014.04.004 (2014).

23. Barr, D. B. et al. Urinary creatinine concentrations in the U.S. population: implications for urinary biologic monitoring measurements. Environ Health Perspect 113, 192-200, doi:10.1289/ehp.7337 (2005).

24. Palermo, M., Shackleton, C. H., Mantero, F. \& Stewart, P. M. Urinary free cortisone and the assessment of 11 beta-hydroxysteroid dehydrogenase activity in man. Clinical endocrinology 45, 605-611 (1996).

25. Steen, N. E. et al. Increased systemic cortisol metabolism in patients with schizophrenia and bipolar disorder: a mechanism for increased stress vulnerability? The Journal of clinical psychiatry 72, 1515-1521, doi:10.4088/JCP.10m06068yel (2011).

26. Yokokawa, A. et al. The effect of water loading on the urinary ratio of cortisone to cortisol in healthy subjects and a new approach to the evaluation of the ratio as an index for in vivo human 11 beta-hydroxysteroid dehydrogenase 2 activity. Steroids 77, 1291-1297, doi:10.1016/j.steroids.2012.07.008 (2012). 
27. Perogamvros, I., Ray, D. W. \& Trainer, P. J. Regulation of cortisol bioavailability-effects on hormone measurement and action. Nat Rev Endocrino/ 8, 717-727, doi:10.1038/nrendo.2012.134 (2012).

28. Ho, J. T. et al. Septic shock and sepsis: a comparison of total and free plasma cortisol levels. The Journal of clinical endocrinology and metabolism 91, 105-114, doi:10.1210/jc.2005-0265 (2006).

29. Dogra, P. \& Vijayashankar, N. P. in StatPearls (2020).

30. Koyama, Y. et al. Free cortisol/cortisone ratio in pooled urine was increased after rapid-ACTH stimulation test under dexamethasone suppression. Endocr J 58, 1099-1103, doi:10.1507/endocrj.ej11-0021 (2011).

31. Jiang, Y. Q. et al. Differential effects of forced swim-stress on the corticotropin-releasing hormone and vasopressin gene transcription in the parvocellular division of the paraventricular nucleus of rat hypothalamus. Neurosci Lett 358, 201-204, doi:10.1016/j.neulet.2004.01.041 (2004).

32. Ma, X. M., Levy, A. \& Lightman, S. L. Emergence of an isolated arginine vasopressin (AVP) response to stress after repeated restraint: a study of both AVP and corticotropin-releasing hormone messenger ribonucleic acid (RNA) and heteronuclear RNA. Endocrinology 138, 4351-4357, doi:10.1210/endo.138.10.5446 (1997).

33. Alfaidy, N., Blot-Chabaud, M., Bonvalet, J. P. \& Farman, N. Vasopressin potentiates mineralocorticoid selectivity by stimulating 11 beta hydroxysteroid deshydrogenase in rat collecting duct. J Clin Invest 100, 2437-2442, doi:10.1172/JCI119785 (1997).

34. Masuzaki, H. et al. A transgenic model of visceral obesity and the metabolic syndrome. Science 294, 21662170, doi:10.1126/science.1066285 (2001).

35. Edwards, C. Sixty years after Hench-corticosteroids and chronic inflammatory disease. The Journal of clinical endocrinology and metabolism 97, 1443-1451, doi:10.1210/jc.2011-2879 (2012).

36. Anagnostis, P. et al. 11 beta-Hydroxysteroid dehydrogenase type 1 inhibitors: novel agents for the treatment of metabolic syndrome and obesity-related disorders? Metabolism: clinical and experimental 62, 21-33, doi:10.1016/j.metabol.2012.05.002 (2013).

37. Zoli, A. et al. ACTH, cortisol and prolactin in active rheumatoid arthritis. Clin Rheumatol 21, 289-293, doi:10.1007/s100670200076 (2002).

38. Wajchenberg, B. L. Subcutaneous and visceral adipose tissue: their relation to the metabolic syndrome. Endocrine reviews 21, 697-738, doi:10.1210/edrv.21.6.0415 (2000).

39. Trumble, S. J. et al. Baleen whale cortisol levels reveal a physiological response to 20th century whaling. Nat Commun 9, 4587, doi:10.1038/s41467-018-07044-w (2018).

40. Soares, M. C., Oliveira, R. F., Ros, A. F., Grutter, A. S. \& Bshary, R. Tactile stimulation lowers stress in fish. Nat Commun 2, 534, doi:10.1038/ncomms1547 (2011).

41. Berger, M. et al. Cortisol Awakening Response and Acute Stress Reactivity in First Nations People. Sci Rep 7, 41760, doi:10.1038/srep41760 (2017).

42. De Marco, R. J., Thiemann, T., Groneberg, A. H., Herget, U. \& Ryu, S. Optogenetically enhanced pituitary corticotroph cell activity post-stress onset causes rapid organizing effects on behaviour. Nat Commun 7, 12620, doi:10.1038/ncomms12620 (2016).

\section{Tables}


Table 1. Distribution of urine markers in morning urine by sex and age.

\begin{tabular}{|c|c|c|c|c|c|c|c|c|c|}
\hline \multirow{2}{*}{ Age (years) } & \multirow[t]{2}{*}{ All } & \multicolumn{4}{|c|}{ Male } & \multicolumn{4}{|c|}{ Female } \\
\hline & & $40-49$ & $50-59$ & $60-69$ & $70-79$ & $40-49$ & $50-59$ & $60-69$ & $70-79$ \\
\hline \multicolumn{10}{|c|}{ Unadjusted cortisol (ng/mL) } \\
\hline Maximum & 493 & 264 & 416 & 245 & 403 & 218 & 295 & 493 & 150 \\
\hline 75 percentile & 35.9 & 40.6 & 41.8 & 37.5 & 35.9 & 36.0 & 34.4 & 34.0 & 33.3 \\
\hline Median & 22.3 & 27.0 & 26.5 & 22.7 & 22.2 & 24.0 & 21.0 & 20.3 & 19.3 \\
\hline 25 percentile & 13.6 & 16.6 & 16.4 & 13.7 & 13.7 & 15.3 & 13.7 & 12.0 & 11.7 \\
\hline Minimum & 0.02 & 0.33 & 0.10 & 0.05 & 0.13 & 0.15 & 0.02 & 0.17 & 0.20 \\
\hline \multicolumn{10}{|c|}{ Unadjusted cortisone (ng/mL) } \\
\hline Maximum & 521 & 387 & 456 & 454 & 417 & 323 & 422 & 521 & 298 \\
\hline 75 percentile & 90.9 & 101 & 108 & 97.5 & 98.4 & 90.1 & 84.2 & 80.5 & 80.1 \\
\hline Median & 62.4 & 72.3 & 74.4 & 69.4 & 68.9 & 61.6 & 57.3 & 55.4 & 54.7 \\
\hline 25 percentile & 42.4 & 49.7 & 50.5 & 48.2 & 46.7 & 42.1 & 39.1 & 37.1 & 37.1 \\
\hline Minimum & 0.28 & 0.63 & 0.28 & 0.87 & 1.09 & 0.52 & 1.77 & 0.93 & 1.36 \\
\hline \multicolumn{10}{|c|}{ Creatinine $(\mathrm{mg} / \mathrm{mL})$} \\
\hline Maximum & 5.45 & 3.81 & 4.09 & 5.45 & 3.31 & 3.99 & 3.60 & 2.85 & 2.08 \\
\hline 75 percentile & 1.31 & 2.00 & 1.69 & 1.48 & 1.35 & 1.55 & 1.17 & 0.90 & 0.78 \\
\hline Median & 0.86 & 1.46 & 1.24 & 1.03 & 0.98 & 1.02 & 0.77 & 0.63 & 0.55 \\
\hline 25 percentile & 0.56 & 0.96 & 0.85 & 0.72 & 0.7 & 0.65 & 0.52 & 0.45 & 0.37 \\
\hline Minimum & 0.08 & 0.14 & 0.17 & 0.09 & 0.15 & 0.09 & 0.10 & 0.08 & 0.12 \\
\hline \multicolumn{10}{|c|}{ Creatinine adjusted cortisol (ng/mg creatinine) } \\
\hline Maximum & 728 & 374 & 277 & 339 & 498 & 159 & 332 & 728 & 387 \\
\hline 75 percentile & 44.5 & 27.4 & 37.6 & 37.6 & 38.1 & 37.9 & 45.3 & 55.1 & 57.6 \\
\hline Median & 25.2 & 18.0 & 20.6 & 21.8 & 22.2 & 23.3 & 26.5 & 30.4 & 33.6 \\
\hline 25 percentile & 15.1 & 11.8 & 13.5 & 12.9 & 13.9 & 13.7 & 16.4 & 18.4 & 20.9 \\
\hline Minimum & 0.03 & 0.33 & 0.06 & 0.05 & 0.30 & 0.25 & 0.03 & 0.22 & 0.85 \\
\hline \multicolumn{10}{|c|}{ Cortisol/cortisone ratio } \\
\hline Maximum & 1.726 & 1.275 & 0.942 & 1.178 & 0.965 & 1.128 & 1.458 & 1.726 & 1.131 \\
\hline 75 percentile & 0.481 & 0.490 & 0.493 & 0.429 & 0.448 & 0.489 & 0.492 & 0.495 & 0.464 \\
\hline Median & 0.358 & 0.359 & 0.372 & 0.323 & 0.319 & 0.381 & 0.377 & 0.369 & 0.348 \\
\hline 25 percentile & 0.262 & 0.267 & 0.265 & 0.239 & 0.231 & 0.281 & 0.277 & 0.273 & 0.259 \\
\hline Minimum & 0.002 & 0.044 & 0.011 & 0.003 & 0.028 & 0.106 & 0.002 & 0.010 & 0.071 \\
\hline
\end{tabular}

Urine samples were collected between 6:00 AM and 11:00 AM.

Table 2. Relationship between biomarker levels in morning urine and participants' characteristics. 
(ng/mg creatinine)

Total

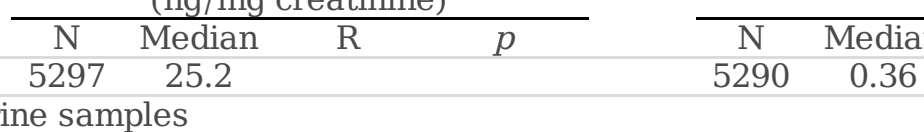

Characteristics of urine samples

Season of collection

\begin{tabular}{|c|c|c|c|c|c|c|c|c|}
\hline Winter & 1467 & 25.52 & -0.032 & 0.0188 & 1467 & 0.331 & 0.090 & $<0.0001$ \\
\hline Autumn/Spring & 2711 & 25.29 & & & 2706 & 0.360 & & \\
\hline Summer & 1119 & 24.48 & & & 1117 & 0.383 & & \\
\hline \multicolumn{9}{|c|}{ nary creatinine $(\mathrm{mg} / \mathrm{mL})$} \\
\hline $0.08-0.65$ & 1593 & 38.28 & -0.403 & $<0.0001$ & 1589 & 0.330 & 0.098 & $<0.0001$ \\
\hline $0.65-1.13$ & 1818 & 26.20 & & & 1817 & 0.352 & & \\
\hline $1.13-5.45$ & 1886 & 18.13 & & & 1884 & 0.388 & & \\
\hline
\end{tabular}

Individual characteristics

Sex

Male

Female

$2182 \quad 21.03$

$3115 \quad 28.33$

$0.176<0.0001$

2178

3112

0.337

$0.091<0.0001$

Age (years)

40-49

50-59

$676 \quad 20.80$

1590

60-69

2170

24.18

70-79

$862 \quad 27.26$

$0.097<0.0001$

$\begin{array}{lll}673 & 0.375 \quad-0.076 & <0.0001\end{array}$

1588

2168

0.374

Menopausal status

$$
\text { Pre }
$$

Peri

$386 \quad 23.97$

$211 \quad 23.38$

$2519 \quad 29.39$

\section{$0.119<0.0001$}

385

210

2517

0.333

Lifestyle habits

Sleep

\begin{tabular}{|c|c|c|c|c|c|c|c|c|}
\hline Regular & 4687 & 25.59 & -0.051 & 0.0002 & 4682 & 0.359 & -0.042 & 0.0022 \\
\hline Irregular & 607 & 23.09 & & & 605 & 0.341 & & \\
\hline \multicolumn{9}{|c|}{ oking (cigarettes/day) } \\
\hline 0 & 4596 & 26.04 & -0.097 & $<0.0001$ & 4591 & 0.360 & -0.043 & 0.0016 \\
\hline$\leq 20$ & 353 & 21.49 & & & 352 & 0.341 & & \\
\hline$>20$ & 347 & 18.43 & & & 346 & 0.322 & & \\
\hline \multicolumn{9}{|c|}{ anol intake ( $\mathrm{g} / \mathrm{kg}$ body weight) } \\
\hline$<0.03$ & 2677 & 26.97 & -0.091 & $<0.0001$ & 2675 & 0.366 & -0.068 & $<0.0001$ \\
\hline$\leq 0.3$ & 1499 & 25.10 & & & 1496 & 0.356 & & \\
\hline$>0.3$ & 1121 & 21.87 & & & 1119 & 0.333 & & \\
\hline \multicolumn{9}{|c|}{ ef corticosteroids } \\
\hline No & 5269 & 25.21 & -0.047 & 0.0007 & 5264 & 0.358 & -0.027 & 0.0472 \\
\hline Yes & 28 & 6.90 & & & 26 & 0.274 & & \\
\hline
\end{tabular}

$\mathrm{R}$

$p$ for difference

Physical activity level*

\begin{tabular}{|lllllllll}
$0.00-1.40$ & 1817 & 24.20 & $\mathbf{0 . 0 5 6}$ & $<0.0001$ & 1814 & 0.347 & $\mathbf{0 . 0 4 5}$ & $\mathbf{0 . 0 0 1 3}$ \\
\hline $1.40-1.47$ & 1772 & 25.66 & & & 1770 & 0.360 & & \\
$1.47-2.24$ & 1626 & 26.17 & & & 1624 & 0.362 & &
\end{tabular}

Perceived stress

Self-reported stress in the last year

$\begin{array}{lllllllll}\text { No } & 1403 & 24.5 & 0.024 & 0.0850 & 1401 & 0.336 & \mathbf{0 . 0 6 6} & <\mathbf{0 . 0 0 0 1} \\ \text { Yes } & 3890 & 25.5 & & & 3885 & 0.364 & & \end{array}$

Physical findings

Systolic blood pressure (mmHg)

\begin{tabular}{|lllllllll}
$82-119$ & 1833 & 24.48 & -0.005 & 0.7236 & 1831 & 0.379 & $-\mathbf{0 . 0 9 1}$ & $<\mathbf{0 . 0 0 0 1}$ \\
\hline $120-136$ & 1724 & 25.75 & & & 1722 & 0.355 & & \\
$137-249$ & 1740 & 25.53 & & & 1737 & 0.336 & & \\
\hline $13.4-21.3$ & 1739 & 27.36 & $-\mathbf{0 . 1 2 9}$ & $\mathbf{< . 0 0 0 1}$ & 1733 & 0.395 & $-\mathbf{0 . 1 6 7}$ & $<\mathbf{0 . 0 0 0 1}$ \\
\hline $21.3-23.8$ & 1794 & 24.91 & & & 1795 & 0.354 & & \\
$23.8-43.7$ & 1757 & 22.91 & & & 1755 & 0.326 & & \\
\hline
\end{tabular}

Laboratory findings

HbA1c (\%)
3.8-5.0
$1737 \quad 24.33$
$0.018 \quad 0.1901$
1737
$0.387-0.135<0.0001$

Page 12/15 


\begin{tabular}{|c|c|c|c|c|c|c|c|c|}
\hline $5.1-5.3$ & 1905 & 25.64 & & & 1902 & 0.356 & & \\
\hline $5.4-14.5$ & 1655 & 25.52 & & & 1651 & 0.328 & & \\
\hline \multicolumn{9}{|l|}{ CRP (mg/dL) } \\
\hline $0.05-0.22$ & 1741 & 26.52 & -0.088 & $<0.0001$ & 1737 & 0.384 & -0.114 & $<0.0001$ \\
\hline $0.22-0.55$ & 1793 & 25.46 & & & 1791 & 0.350 & & \\
\hline $0.55-117$ & 1763 & 23.41 & & & 1762 & 0.334 & & \\
\hline
\end{tabular}

Urine samples were collected between 6:00 AM and 11:00 AM. R, Spearman's rank correlation coefficient; HbA1c, hemoglobin A1C; CRP, C-reactive protein. *the ratio of total energy expenditure to basal metabolic rate. There are missing values in some categories, including the following: sleep regularity (3), smoking (1), physical activity level (82), perceived stress (4), blood pressure (7), and BMI (7).

Table 3. Odds ratio of physical and clinical characteristics associated with poor health or disease histories by cortisol markers.

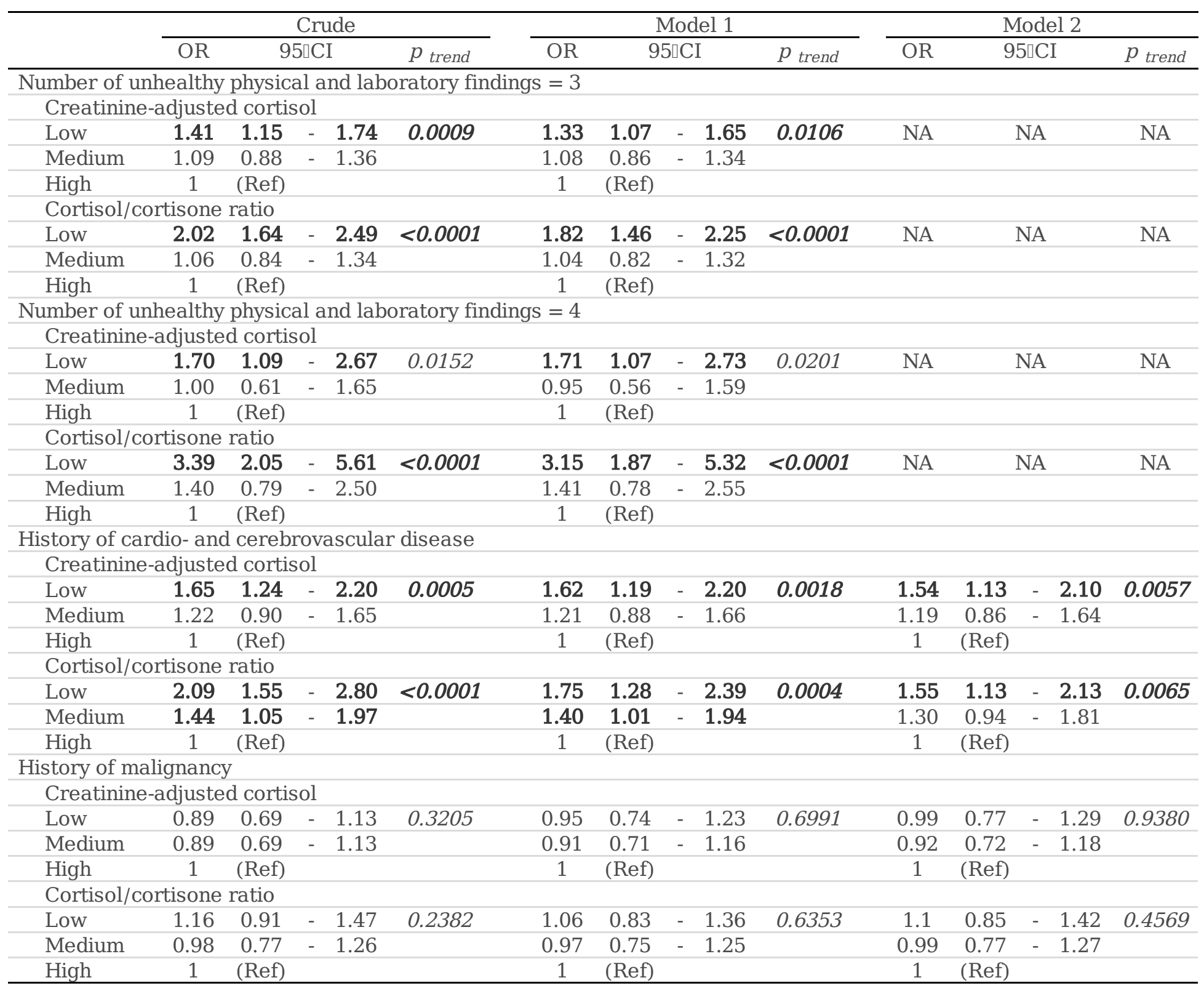


Physical and clinical criteria for poor health were defined by a BMI $\geq 25$, systolic blood pressure $\geq 140 \mathrm{mmHg}, \mathrm{CRP} \geq 0.5$ $\mathrm{mg} / \mathrm{dL}$, and HbA1c $\geq 5.6 \%$. Model 1 includes covariates of sex, age, smoking and drinking, physical activity, use of corticosteroids, and perceived stress. Model 2 includes covariates as in Model 1 plus systolic blood pressure, BMI, hemoglobin A1c and C-reactive protein. Urine samples were collected between 6:00 AM and 11:00 AM. Levels of cortisol were divided into tertiles (low, medium, high). OR, odds ratio; CI, confidence interval; $\mathrm{p}_{\text {trend, }} \mathrm{p}$ value for trend (low $=0$, medium =1, high =2); NA, not assessed.

\section{Figures}

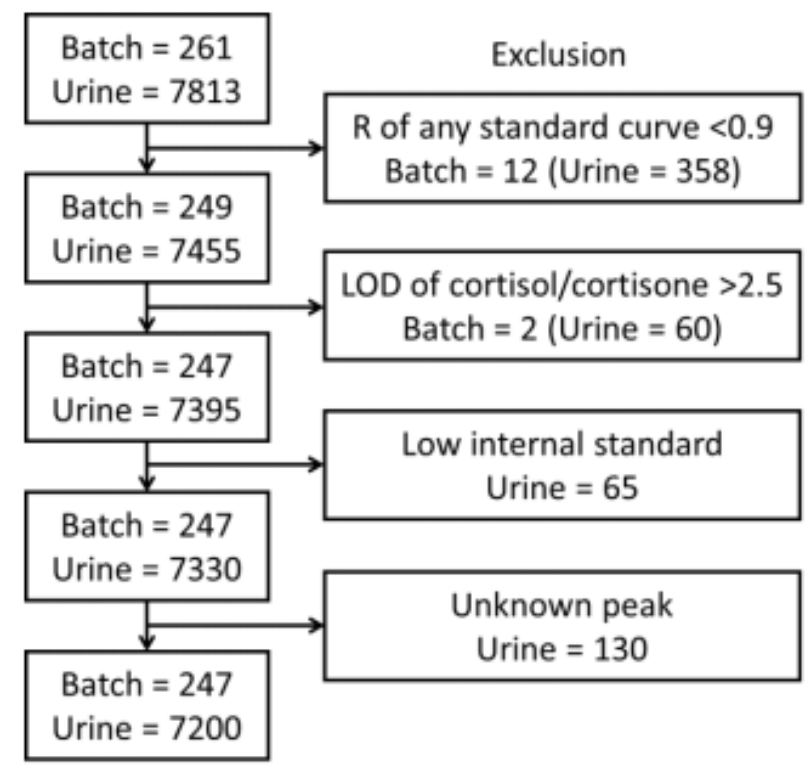

\section{Figure 1}

Flow diagram. During the 15-month analysis period, careful quality control was performed by monitoring fluctuations in the mass spectrometer, matrix effects, and other factors. Of the 7813 urine samples, including 5717 morning urine collected between 6:00 AM and 11:00 AM, 613 urine samples were quantified with insufficient accuracy and excluded from our study. A batch comprises 30 or fewer unknown samples and 16 standards; R, correlation coefficient; LOD, lower limit of detection. 
A

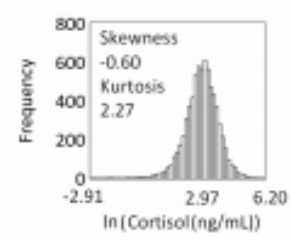

B

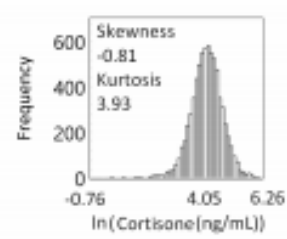

c

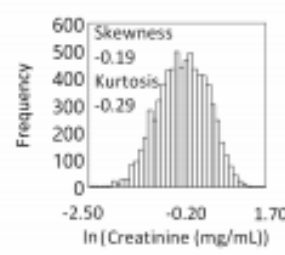

D

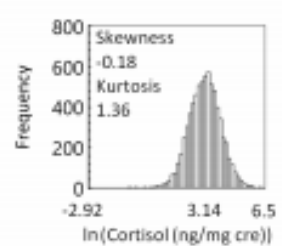

E

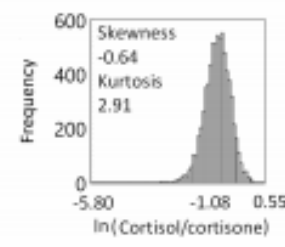

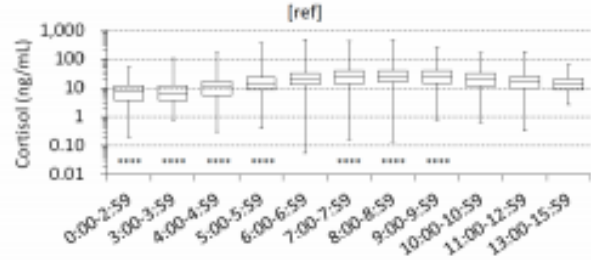
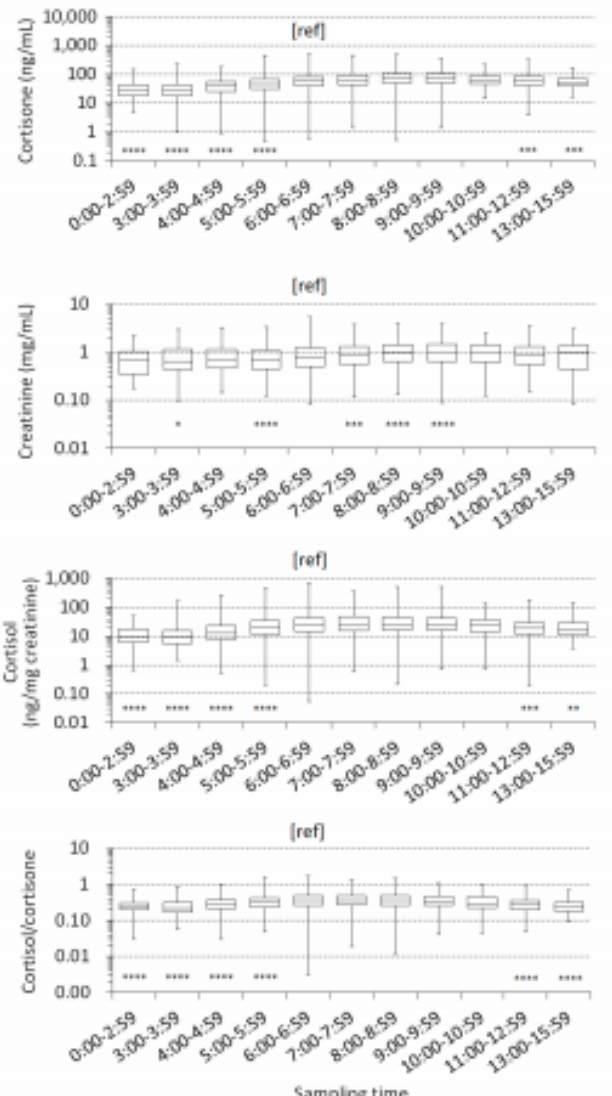

\section{Figure 2}

Distribution of urinary markers. Histograms of free cortisol $(A, n=7200)$, free cortisone $(B, n=7200)$, creatinine $(C, n=7199)$, creatinine-adjusted free cortisol $(D, n=7199)$ and the ratio of free cortisol to cortisone $(E, n=7186)$ with minimum, median and maximum values presented in boxplots by collection time. Dunnett's tests on log scales were performed to compare hormone levels with urine collection times (reference, 6:00-6:59; *** $p$ $\left.<0.0001,{ }^{* \star *} p<0.001, * * p<0.01, * p<0.05\right)$.

\section{Supplementary Files}

This is a list of supplementary files associated with this preprint. Click to download.

- SIAppendix.pdf 